\title{
Impact of uncertainties in cardiac mechanics simulations
}

\author{
Joventino O. Campos ${ }^{1,2}$, Joakim Sundnes ${ }^{3}$, Rodrigo W. dos Santos ${ }^{1}$, Bernardo M. Rocha ${ }^{1}$ \\ ${ }^{1}$ Programa de Pós-Graduação em Modelagem Computacional \\ Universidade Federal de Juiz de Fora (UFJF) \\ CEP 36036-330 - Juiz de Fora - MG - Brasil \\ ${ }^{2}$ Centro Federal de Educação Tecnológica de Minas Gerais (CEFET-MG) \\ CEP 36700-000 - Leopoldina - MG - Brasil \\ ${ }^{3}$ Simula Research Laboratory - Oslo, Noruega \\ \{jcampos, bernardo.rocha\}@ice.ufjf.br, sundnes@simula.no, rodrigo.weber@ufjf.edu.br
}

\begin{abstract}
The heart simulations have a high potential for use in diagnosis and therapy planning. However, such clinical use puts strict demands on the reliability of the model predictions due to errors and uncertainty in the model inputs. The model parameters are difficult to be measured and they are associated with considerable uncertainty. Furthermore, patient-specific geometries are generated from medical images involving semi-manual processing, which becomes a potential source of uncertainty. In order to contribute to the reliability assessment of cardiac mechanics models, in this study we apply uncertainty quantification and sensitivity analysis for finite element simulations of the left ventricle during the cardiac cycle.
\end{abstract}

Resumo. As simulações do coração têm grande potencial de aplicações em diagnósticos e planejamento de terapias. Porém, tal uso clínico precisa conhecer a confiabilidade das predições devido a erros e incertezas nas entradas destes modelos. Os parâmetros do modelo são difíceis de ser medidos e estão associados a uma incerteza considerável. Além disso, geometrias para pacientes específicos são geradas a partir de imagens médicas envolvendo algum processamento manual, o que as tornam uma potencial fonte de incertezas. Com o objetivo de contribuir para a avaliação da confiabilidade de modelos da mecânica cardíaca, neste estudo foram realizadas análises de quantificação de incertezas e sensibilidade de simulações do ventrículo esquerdo durante o ciclo cardíaco.

\section{Introdução}

Modelos computacionais que reproduzem o comportamento eletromecânico do coração têm sido uma importante ferramenta para pesquisas na área médica. Além disso, o desenvolvimento de simulações personalizadas por paciente para uso em aplicações clínicas é um tema de grande interesse [Arevalo et al. 2016]. Este tipo de simulação requer a construção de uma geometria a partir de imagens médicas e o ajuste de vários parâmetros do modelo. Tais processos possuem várias fontes de incerteza que podem impactar nas predições do modelo e, por isso, é importante verificar o impacto das mesmas nos resultados das simulações. 
A construção de geometrias personalizadas geralmente envolve um processo de segmentação semi-manual a partir de imagens de ressonância magnética, onde os contornos do endocárdio e epicárdio precisam ser marcados com o auxílio de especialistas, o que acaba sendo uma grande fonte de incerteza. O estudo [Suinesiaputra et al. 2014] mostra a variação encontrada quando diferentes especialistas realizam a marcação destes contornos e como isso impacta na espessura da parede do ventrículo e em outras medidas clínicas. A orientação de fibras do tecido cardíaco também pode ser obtida a partir de imagens médicas com uma precisão de $\pm 10^{\circ}$ [Reese et al. 1995], que é uma variabilidade significativa.

Trabalhos anteriores tem estudado o impacto de incertezas em simulações da mecânica cardíaca. O estudo de [Rodríguez-Cantano et al. 2019], por exemplo, analisou o impacto de incertezas da orientação de fibras e propriedades do tecido cardíaco em simulações da mecânica cardíaca durante a fase de preenchimento do ventrículo esquerdo.

O presente trabalho tem o objetivo de contribuir com o estado da arte de modelos cardíacos apresentando uma análise de sensibilidade e quantificação de incertezas de simulações da mecânica do ventrículo considerando o ciclo cardíaco completo. Incertezas são consideradas nos parâmetros do material, na orientação de fibras e na geometria do ventrículo esquerdo, avaliando o impacto destas incertezas em quantidades de interesse clínico.

\section{Caracterização do problema}

A quantificação de incertezas tem o objetivo de determinar como incertezas nas entradas são propagadas para suas predições. As entradas do modelo podem ser parâmetros das equações consideradas, condições de contorno ou propriedades da geometria. Para incorporar incertezas nestas entradas, as técnicas de quantificação de incerteza consideram variáveis aleatórias contínuas descritas por uma função de distribuição de probabilidade, como mostra a Figura 1 (a). Então amostras destas distribuições são geradas e o modelo determinístico é avaliado para um conjunto de amostras, com o objetivo de calcular medidas estatísticas de algumas quantidades de interesse, possibilitando a avaliação de sua variabilidade (Figura $1(\mathrm{~d})$ ).

A análise de sensibilidade é usada para avaliar a contribuição de uma entrada em alguma quantidade de interesse. Para realizar esta análise, o método dos índices Sobol foi utilizado [Campos et al. 2019]. O índice Sobol principal $S_{m}^{i}$ é usado para identificar entradas do modelo que têm alto impacto em quantidades de interesse, as quais deveriam ser medidas ou estimadas com maior precisão (Figura 1 (e)). Já o índice Sobol total $S_{t}^{i}$ é usado para identificar entradas que têm baixa influência nas quantidades de interesse, as quais possuem $S_{t}^{i} \approx 0$.

O modelo determinístico descreve a deformação do tecido cardíaco através da equação de equilíbrio de Cauchy, a qual é resolvida para se encontrar o campo de deslocamentos $\mathbf{u}$ devido a algum carregamento (Figura 1 (b)). O tensor de tensões $\sigma$ é derivado de um modelo constitutivo, que relaciona tensão e deformação para um determinado material. O modelo constitutivo usado para o tecido cardíaco foi o proposto por [Guccione et al. 1991], que descreve o comportamento deste material na presença de uma carga. A contração cardíaca é desenvolvida por uma força ativa, gerada no nível celular, que foi representada neste trabalho pelo modelo celular eletromecânico apresentado 


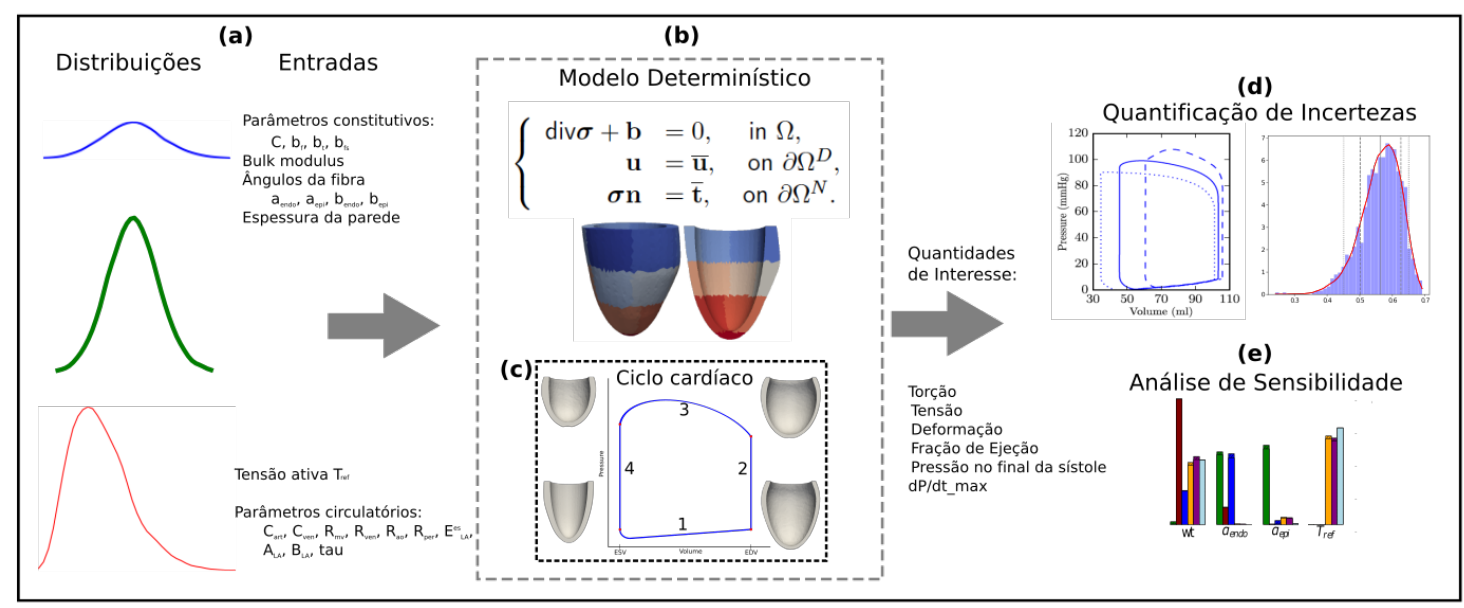

Figura 1. Análise de quantificação de incertezas e sensibilidade para simulações da atividade do ventrículo esquerdo durante o ciclo cardíaco. Distribuições de probabilidade são assumidas para diferentes entradas do modelo e então medidas estatísticas são avaliadas para as quantidades de interesse extraídas das predições.

em [Kerckhoffs et al. 2003]. O método dos elementos finitos usando uma formulação variacional mista, como descrito em [Campos et al. 2017], é utilizado para resolver este modelo da mecânica cardíaca.

As simulações realizadas consideraram geometrias do ventrículo esquerdo que podem ser personalizadas por paciente. Estas geometrias foram construídas através de um algoritmo baseado no diagrama AHA de 17 segmentos [Cerqueira et al. 2002], que divide o ventrículo esquerdo em 6 segmentos para a região basal, 6 para a região medial, 4 para a região apical e 1 segmento para o ápice, como apresentado na Figura 2 (a). Para criar a geometria, o algoritmo em questão utiliza uma parametrização elipsoidal através de informações da espessura da parede do ventrículo nestes 17 segmentos, além do diâmetro da base no eixo curto e o comprimento ápice-base no eixo longo, como apresentado em [Campos et al. 2019].

A orientação de fibras foi atribuída à malha de elementos finitos através de um procedimento desenvolvido por [Bayer et al. 2012]. Nesta abordagem, a direção longitudinal da fibra gira no sentido horário de um ângulo $\alpha_{\text {endo }}$ na superfície do endocárdio até um ângulo $\alpha_{e p i}$ na superfície do epicárdio. A direção transversal da fibra é considerada perpendicular à longitudinal e varia de $\beta_{\text {endo }}$ a $\beta_{\text {epi }}$. A Figura 2 (c) apresenta um exemplo de orientação de fibras, com ângulos: $\alpha_{\text {endo }}=60^{\circ}, \alpha_{\text {epi }}=-60^{\circ}, \beta_{\text {endo }}=65^{\circ} \mathrm{e}$ $\beta_{\text {epi }}=25^{\circ}$.

As condições de contorno neste problema foram os descolamentos prescritos na base do ventrículo e a pressão aplicada na superfície do endocárdio. Todos os pontos da base do ventrículo foram restringidos de se deslocar nas direções $x$ e $y$, enquanto os pontos da base referentes ao epicárdio também foram impedidos de se deslocar na direção $z$, como realizado em [Campos et al. 2019]. A superfície do endocárdio está sujeita a uma pressão exercida pelo sangue que entra e sai da cavidade do ventrículo. Para representar este funcionamento foi utilizada uma condição de contorno de pressão que varia no tempo, através de um modelo do tipo Windkessel, apresentado em [Shavik et al. 2017], 


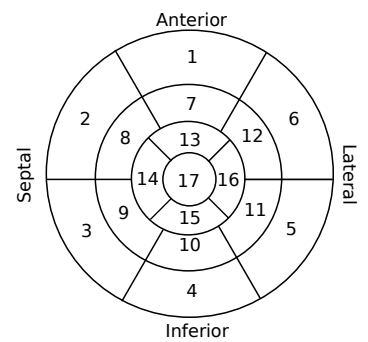

(a)

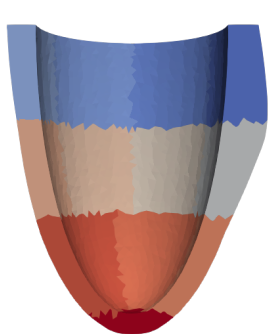

(b)

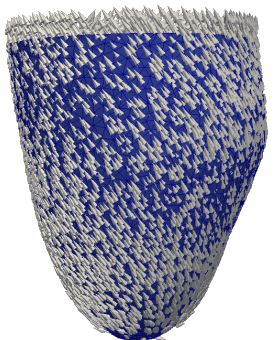

(c)

Figura 2. Geometria do ventrículo esquerdo. (a) Diagrama AHA de 17 segmentos. (b) Corte da geometria gerada pelo algoritmo com a marcação dos blocos correspondentes a cada segmento. (c) Orientação de fibras atribuída à malha do ventrículo.

que representa o ciclo completo do ventrículo (Figura 1 (c)).

\section{Experimento}

Esta seção apresenta os resultados de um dos experimentos desenvolvidos na tese e publicado no trabalho [Campos et al. 2020]. As análises de quantificação de incertezas e sensibilidade foram realizadas considerando incertezas em parâmetros do modelo constitutivo, na espessura da parede do ventrículo, nos ângulos de orientação da fibra e na magnitude da tensão ativa, os quais são regidos por distribuições Normais, com coeficiente de variação de 5\%. O coeficiente de variação (COV) é a razão entre o desvio padrão e a média. A incerteza na espessura da parede foi considerada utilizando uma única variável aleatória, assim toda vez que uma amostra para esta variável foi gerada, seu valor foi utilizado para variar cada seguimento do diagrama AHA apresentado na Figura 2 (a). Portanto, foram criadas amostras de geometrias onde a espessura da parede varia ao longo do ventrículo esquerdo.

Foram definidas algumas quantidades de interesse relacionadas à deformação do tecido cardíaco: torção, tensão e deformação na região medial do ventrículo, que foram medidas no início da fase de ejeção. Além de outras quantidades globais usadas por médicos para verificar o comportamento do coração, que podem ser extraídas da curva pressão-volume, como a apresentada na Figura 1 (c). Estas quantidades foram a fração de ejeção $E F=\frac{E D V-E S V}{E D V}$, calculada usando o volume no final da diástole (EDV) e o volume no final da sístole (ESV), ambos representados na Figura 1 (c). Além da pressão também no final da sístole (ESP) e a variação máxima da pressão com relação ao tempo $d P / d t_{\max }$.

Após o cálculo das quantidades de interesse e geração do polinômio, a propagação de incerteza foi verificada através da média, desvio padrão e coeficiente de variação dos resultados, como apresenta a Tabela 1 . Pode-se notar que a torção foi altamente impactada pelas incertezas consideradas, com uma variação de $16 \%$ em relação à média. Além da torção, a fração de ejeção também teve uma variação significativa de $13.4 \%$, enquanto as demais quantidades tiveram coeficiente de variação próximo ao coeficiente de $5 \%$ definido na entrada. A fração de ejeção também teve uma variação significativa, próximo à variação definida na entrada.

Para verificar a influência de cada entrada na variabilidade encontrada para as 
Tabela 1. Média, desvio padrão (STD) e coeficiente de variação (COV) das quantidades de interesse (Qol).

\begin{tabular}{l|rrr|rrr}
\hline QoI & Torsion & $\sigma_{f}$ & $e_{f}$ & $\mathrm{ESP}$ & $\mathrm{EF}$ & $d P / d t_{\max }$ \\
\hline Média & 9.92 & 102.32 & 0.192 & 94.00 & 0.56 & 1104.47 \\
STD & 1.58 & 7.36 & 0.011 & 3.94 & 0.07 & 71.94 \\
COV & 16.0 & 7.20 & 5.6 & 4.2 & 13.4 & 6.5 \\
\hline
\end{tabular}

quantidades de interesse, foram calculados os índices Sobol apresentados na Figura 3, na qual para cada parâmetro são apresentadas seis barras coloridas, onde cada cor representa uma das quantidades de interesse. Os resultados mostram que as quantidades são fortemente impactadas por variações na espessura da parede do ventrículo, enquanto os parâmetros do modelo constitutivo causam pouca alteração nestas saídas. A magnitude da tensão ativa $T_{r e f}$ também tem influência significativa nas medidas extraídas do diagrama pressão-volume: pressão no final da sístole, fração de ejeção e $d P / d t_{\text {max }}$. Além disso, variações nos ângulos das fibras afetam as medidas de torçao e deformação.

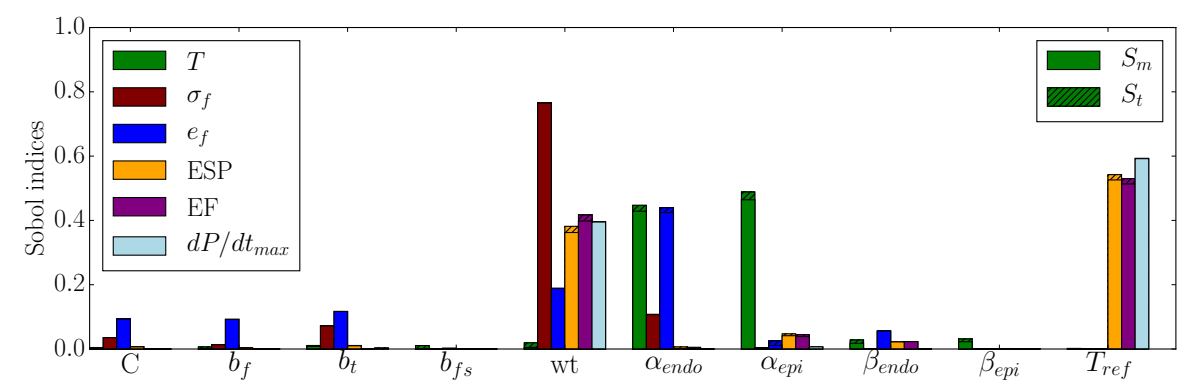

Figura 3. Análise de sensibildiade através dos índices Sobol principal (barras sólidas) e total (barras tracejadas).

\section{Conclusão}

O presente trabalho apresentou a quantificação de incertezas e análise de sensibilidade para simulações da mecânica cardíaca que reproduzem o ciclo completo do ventrículo esquerdo. Foram consideradas incertezas nos parâmetros constitutivos, na orientação de fibra, na geometria e na tensão ativa. Uma abordagem baseada no diagrama AHA de 17 segmentos e na parametrização elipsoidal foi utilizada para gerar as geometrias do ventrículo de forma personalizada, que também possibilita considerar incertezas em diferentes regiões. Os resultados mostraram que a espessura da parede e a magnitude da tensão ativa são as fontes de incertezas que mais influenciam nas quantidades de interesse escolhidas. Particularmente, a espessura da parede domina a influência na tensão, um importante indicador na verificação de algumas doenças cardíacas que é difícil de ser medida e geralmente é calculada via simulações personalizadas por paciente. Outra quantidade importante no diagnóstico de doenças é a fração de ejeção, que também foi bastante influenciada por estas fontes de incerteza. Além disso, a torção foi bastante influenciada pelas incertezas na orientação de fibras. Portanto, este estudo demonstra a necessidade de precisão na geração das geometrias, orientação de fibras e na escolha da tensão ativa utilizadas em simulações personalizadas por paciente, para que estas possam ser traduzidas em aplicações clínicas. 


\section{Referências}

Arevalo, H. J., Vadakkumpadan, F., Guallar, E., Jebb, A., Malamas, P., Wu, K. C., and Trayanova, N. A. (2016). Arrhythmia risk stratification of patients after myocardial infarction using personalized heart models. Nature Com, 7.

Bayer, J. D., Blake, R. C., Plank, G., and Trayanova, N. A. (2012). A novel rule-based algorithm for assigning myocardial fiber orientation to computational heart models. Annals of biomedical engineering, 40(10):2243-2254.

Campos, J., Sundnes, J., Dos, R. S., and Rocha, B. (2019). Effects of left ventricle wall thickness uncertainties on cardiac mechanics. Biomechanics and modeling in mechanobiology, pages 1-13.

Campos, J., Sundnes, J., Dos, R. S., and Rocha, B. (2020). Uncertainty quantification and sensitivity analysis of left ventricular function during the full cardiac cycle. Philosophical Transactions A, 20190381:1-19.

Campos, J. O., Santos, R. W., Sundnes, J., and Rocha, B. M. (2017). Preconditioned augmented lagrangian formulation for nearly incompressible cardiac mechanics. International Journal for Numerical Methods in Biomedical Engineering, 34(4):e2948. e2948 cnm.2948.

Cerqueira, M. D., Weissman, N. J., Dilsizian, V., Jacobs, A. K., Kaul, S., Laskey, W. K., Pennell, D. J., Rumberger, J. A., Ryan, T., Verani, M. S., et al. (2002). Standardized myocardial segmentation and nomenclature for tomographic imaging of the heart. Circulation, 105(4):539-542.

Guccione, J. M., McCulloch, A. D., Waldman, L., et al. (1991). Passive material properties of intact ventricular myocardium determined from a cylindrical model. $J$ Biomech Eng, 113(1):42-55.

Kerckhoffs, R., Bovendeerd, P., Kotte, J., Prinzen, F., Smits, K., and Arts, T. (2003). Homogeneity of cardiac contraction despite physiological asynchrony of depolarization: a model study. Annals of biomedical engineering, 31(5):536-547.

Reese, T. G., Weisskoff, R. M., Smith, R. N., Rosen, B. R., Dinsmore, R. E., and Wedeen, V. J. (1995). Imaging myocardial fiber architecture in vivo with magnetic resonance. Magnetic Resonance in Medicine, 34(6):786-791.

Rodríguez-Cantano, R., Sundnes, J., and Rognes, M. E. (2019). Uncertainty in cardiac myofiber orientation and stiffnesses dominate the variability of left ventricle deformation response. International journal for numerical methods in engineering, 79(11):120 .

Shavik, S. M., Wall, S. T., Sundnes, J., Burkhoff, D., and Lee, L. C. (2017). Organ-level validation of a cross-bridge cycling descriptor in a left ventricular finite element model: effects of ventricular loading on myocardial strains. Physiological reports, 5(21).

Suinesiaputra, A., Cowan, B. R., Al-Agamy, A. O., Elattar, M. A., Ayache, N., Fahmy, A. S., Khalifa, A. M., Medrano-Gracia, P., Jolly, M.-P., Kadish, A. H., et al. (2014). A collaborative resource to build consensus for automated left ventricular segmentation of cardiac mr images. Medical image analysis, 18(1):50-62. 